\title{
Enhanced ultrafiltration PES membranes doped with mesostructured functionalized silica particles
}

\author{
Antonio Martín a , Jesús M. Arsuaga a , Nuria Roldán a , Javier de Abajo ${ }^{\mathrm{b}}$, Ana Martínez ${ }^{\mathrm{c}}$, Arcadio Sotto $^{\mathrm{a}, *}$ \\ a Department of Chemical and Environmental Technology, Rey Juan Carlos University, Madrid, Spain \\ b Department of Macromolecular Chemistry, Institute of Polymer Science and Technology, CSIC, 28006 Madrid, Spain \\ c Department of Basic Sciences Applied to Engineering, Technical University of Madrid, Madrid, Spain
}

\section{H I G H L I G H T S}

- Mesostructured silica functionalized with amino or carboxylic groups was synthesized.

- Novel PES composite membranes were prepared by doping with mesostructured SBA-15.

- Membrane morphology was determined by accessibility and number of hydrophilic sites.

- Enhanced hydrophilicity and permeation are gained for PES/mesostructured SBA-15.

- Antifouling membrane properties are increased by doping mesostructured SBA-15.

\section{G R A P H I C A L A B S T R A C T}

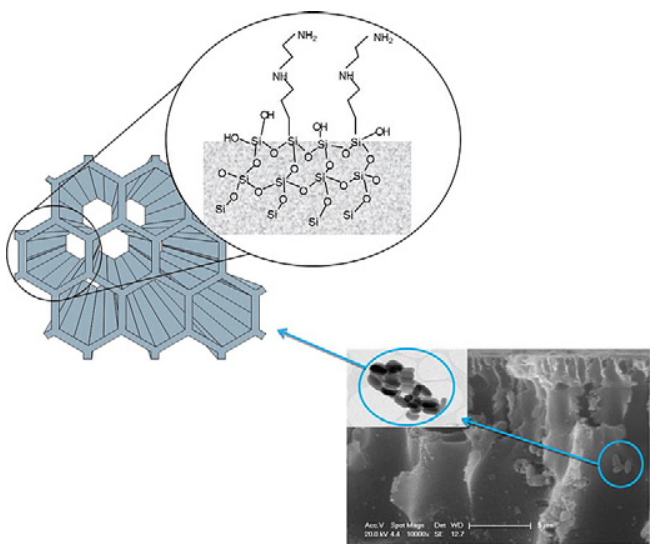

\section{A B S T R A C T}

Novel hybrid ultrafiltration membranes have been prepared by incorporation of mesostructured functionalized silica particles in polyethersulfone. Physico-chemical characterization of synthesized materials was accomplished to determine the textural and structural properties of particles by nitrogen adsorption-desorption and TEM. A successful functionalization of silica with amine and carboxylic groups was confirmed by means of MAS-NMR. Membrane surface morphology was studied in terms of pore size distribution, porosity and hydrophilicity, suggesting a great influence of mesostructured silica incorporation in polyethersulfone on the polymer matrix configuration. Membrane functions were significantly improved as a result of a considerable increase of water permeation without affecting negatively the membrane selectivity. The antifouling membrane properties were also enhanced, especially against irreversible fouling. Multi-run fouling tests of modified membranes confirmed the stability of permeation through membranes doped with mesostructured functionalized silica particles.

(C) 2014 Elsevier B.V. All rights reserved.

\footnotetext{
* Corresponding author.
}

\section{Introduction}

Ultrafiltration (UF) is one of the most common membrane separation processes applied to water treatment, specifically to retain desirable and undesirable water components such as proteins, viruses, 
colloids and pathogens [1,2]. However, because of the hydrophobic nature of most polymers used for UF membrane preparation, these filtration materials are susceptible to organic fouling due to the adsorption or deposition of retained organic compounds [3,4]. In this regard, polyethersulfone (PES) appears quite suitable for this application due to its high chemical and thermal stabilities, good oxidation resistance and excellent mechanical properties [5]. In comparison with other organic polymers, like polysulfone (PSf), PES exhibits higher hydrophilicity, which can increase the permeation and antifouling properties of the UF membrane.

Incorporation of inorganic particles into membranes, related to modification of polymeric materials via improving its hydrophilic character, has been proven to successfully reduce the membrane fouling [6-8]. Addition of selected hydrophilic particles in the membrane matrix increases the polymer hydrophilicity and enhances the water permeability while mitigating the membrane fouling $[9,10]$. Many types of nanomaterials have been tested as membrane fillers for this purpose in the last years, including titania $\left(\mathrm{TiO}_{2}\right)[9,10]$, zirconia $\left(\mathrm{ZrO}_{2}\right)$ [11], silica $\left(\mathrm{SiO}_{2}\right)$ [12], zinc oxide $(\mathrm{ZnO})$ [13], alumina $\left(\mathrm{Al}_{2} \mathrm{O}_{3}\right)$ [14], silver $(\mathrm{Ag})$ [15] and carbon nanotubes [16].

Morphological and permeation properties of the modified membranes are very sensitive to the weight ratio of the incorporated particles. Thus, an excessive amount of fillers could lead to particle aggregation declining the dispersion grade; moreover, membrane permeation is not always enhanced due to the risk of pore blocking. Therefore, Sotto et al. proposed a relatively low particle concentration (less than 1\%) to avoid this adverse effect [17]. This strategy has been adopted in this work.

Among the potential filler materials, $\mathrm{SiO}_{2}$ has been extensively studied for modification of membranes because of its excellent chemical stability and high hydrophilicity [18-22]. However, most of the previous studies have incorporated non-ordered mesoporous silica particles at concentration values higher than 0.75 wt.\%. Conversely, the addition of mesostructured functionalized silica particles at lower concentration into the PES matrix is proposed in this work to explore two effects: porous ordering and silica functionalization.

Mesostructured silica particles have been widely applied in the areas of catalysis, adsorption and separation processes [23]. In particular, SBA-15 has been one of the most extensively studied materials due to its chemical, thermal and mechanical stability, large pore size with narrow pore size distribution, high surface area and easy surface functionalization. A high number of different functionalities have been incorporated onto the SBA-15 material [24]. Two of the most attractive functionalities are amino [25-29] and carboxylic acid groups [30,31]. There are two main approaches of silica surface functionalization; post-synthesis grafting, which is based on the reaction between an organoalkoxysilane precursor of the organic chain and the silanol groups of the silica support and co-condensation method, that involves the simultaneous condensation in the synthesis medium of the organoalkoxysilane compound and the alkoxysilane precursor of the silica structure [32]. However, certain drawbacks are associated with post-synthesis grafting. Thus, this method usually results in inhomogeneous surface coverage due to preferential organic moiety congregation near the entries of the mesopores and the exterior surface [33]. In addition, a restricted loading level of functional groups can be grafted because of the limited density of silanol groups at the reactive surface [34].

A new approach of particle incorporation into the polymer matrix is proposed in this study by adding co-condensation functionalized SBA-15 particles at rather low concentration. The main goal of this research is to evaluate the effect of functionalized mesostructured silica fillers on the membrane morphology and performance of doped PES membranes. Also, the aim of this paper is to investigate the influence of different variables of the porous silica particles such as the porous ordering, functionalization with amino and carboxylic groups and degree of accessibility to the permeation media of the organic groups incorporated into silica. In addition, the effect of added particle concentration on the membrane performance will be explored.

\section{Materials and methods}

\subsection{Synthesis of pure silica SBA-15}

Conventional SBA-15 silica was synthesized according to an already described method [35]. Usually, $4 \mathrm{~g}$ of the structure-directing agent block-copolymer Pluronic $123\left(\mathrm{EO}_{20} \mathrm{PO}_{70} \mathrm{EO}_{20}\right.$, Mn 5800, Aldrich) was dissolved with stirring in $1.9 \mathrm{M} \mathrm{HCl}(125 \mathrm{~mL})$ at room temperature. The resultant micellar solution was then heated to $40{ }^{\circ} \mathrm{C}$ before adding the silica source, tetraethylorthosilicate (TEOS, Aldrich). After being stirred for $20 \mathrm{~h}$ at $40{ }^{\circ} \mathrm{C}$, the mixture was aged under static conditions and autogenous pressure at $100^{\circ} \mathrm{C}$ for $24 \mathrm{~h}$. Thereafter, the solid product was recovered by filtration and air dried overnight. The surfactant was removed by calcination at $550{ }^{\circ} \mathrm{C}$ for $5 \mathrm{~h}\left(1.8^{\circ} \mathrm{C} / \mathrm{min}\right.$ heating ramp).

\subsection{Direct synthesis of organosilane-functionalized SBA-15 samples}

The synthesis of the different organically functionalized SBA-15 particles was quite similar to that of the conventional SBA-15 except for adding a certain amount of selected organosilanes $1 \mathrm{~h}$ after adding the silica source (TEOS). In addition, surfactant extraction was accomplished by ethanol-washing reflux instead of calcination. Thus, in the case of the amino functionalized silica materials, [3-(2-aminoethylamino) propyl] trimethoxysilane (AEAPTMS, Aldrich) and (3-aminopropyl) trimethoxysilane (APTMS, Aldrich) were used as organosilane with an organosilane/TEOS molar ratio of $15 \%$. In the case of the carboxylic functionalized silica materials, carboxyethylsilanetriol sodium salt (CES, ABCR) was used as carboxylic group source with organosilane/TEOS molar ratios of $15 \%$ and $30 \%$. The resultant materials were denoted as SBA-15/AEAPTMS-15, SBA-15/APTMS-15, SBA-15/CES-15 and SBA-15/ CES-30 respectively (Fig. 1 ).

\subsection{Silica particle characterization}

The synthesized silica particles were structurally characterized by nitrogen adsorption-desorption and transmission electron microscopy (TEM). Nitrogen adsorption-desorption isotherms at $77 \mathrm{~K}$ were determined using a Micromeritics TRISTAR 3000 system. The data were analyzed using the BJH and BET models and the pore total volume (Vp) was assigned at $\mathrm{P} / \mathrm{Po}=0.975$ as single point. TEM images were acquired in a PHILIPS TECNAI-20 electronic microscope operating at $200 \mathrm{kV}$.

Nitrogen content was determined by means of elemental analysis (HCNS) in a Vario EL III apparatus. ${ }^{29} \mathrm{Si}$ solid-state nuclear magnetic resonance (NMR) was used to characterize the environment of the silicon nuclei. NMR measurements were conducted at room temperature with a magnetic field strength of $9.4 \mathrm{~T}$ on a high-resolution Varian INFINITY PLUS 400 NMR spectrometer operating at $79.5 \mathrm{MHz}$. Solid-state single-pulse ${ }^{29} \mathrm{Si}$ MAS-NMR spectra were recorded using a $1.5 \mu \mathrm{s} \pi / 2$ pulse, a recycle delay of $20 \mathrm{~s}$ and 3000 transients.

To determine the particle size distribution a MasterSizer Laser Diffraction Particle Size Analyzer (Malvern Instrument Ltd., Malvern, England) was used. Selected particles dispersed in 1-methyl-2pyrrolidone (NMP) were sonicated for 15 min using a bath sonicator ( 40 W, 50 Hz, Fisher Scientific Fair Lawn, New Jersey) before the size measurement. Repeatability of all hydrodynamic sizes was verified with more than four measurements.

\subsection{Membrane preparation}

Polyethersulfone (PES, $58 \mathrm{kDa}$ ) provided by BASF Co. (Germany) was used for preparation of polymeric membranes. This polymer was dried at $110^{\circ} \mathrm{C}$ in an oven overnight prior to use for the casting solution preparation. PES was dissolved in NMP solvent purchased from Sigma Chemical Co. Ltd (St. Louis, USA). The non-woven support layer AWA18 was obtained from Freudenberg (Weinheim, Germany). The doped membranes were prepared by fully dispersing 0.6 and $1 \mathrm{wt} . \%$ of fillers 


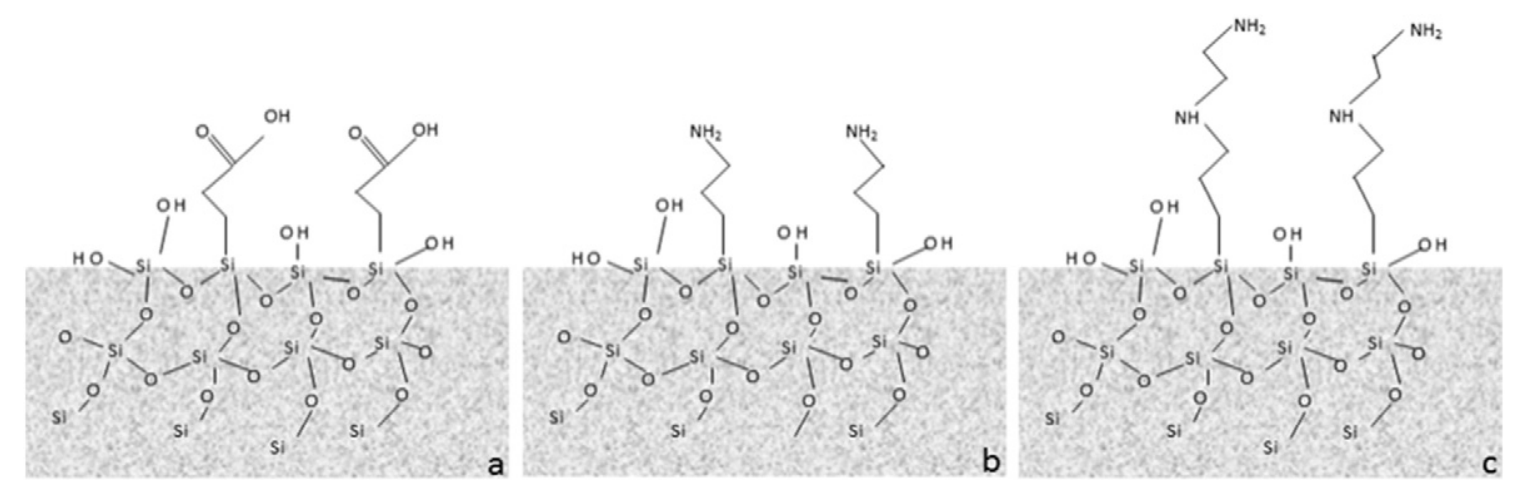

Fig. 1. Scheme showing the simplified chemical structures of synthesized particles. (a) SBA-15/CES-15 and 30. (b) SBA-15/APTMS-15. (c) SBA-15/AEAPTMS-15.

in the corresponding volume of NMP for $3 \mathrm{~h}$ by mechanical stirring at $300 \mathrm{rpm}$ and sonicated for $1 \mathrm{~h}$. Subsequently, the PES polymer was added to the solution and stirred for $24 \mathrm{~h}$ at $600 \mathrm{rpm}$ and room temperature. Afterwards, the air bubbles that might be trapped in the polymer solution were removed. The composition of the prepared membranes (denoted as $0, \mathrm{~A}-\mathrm{F}$ ) is summarized in Table 1.

The polymeric solutions were cast on a glass plate with non-woven polyester support with initial thickness of $200 \mu \mathrm{m}$ by a filmograph (K4340 Automatic Film Applicator, Elcometer) to obtain a defect-free membrane. The cast films were then immersed in a nonsolvent coagulation bath (distilled water) for precipitation. After $15 \mathrm{~min}$, the obtained membranes were repeatedly washed with distilled water to remove the remaining solvent and stored in distilled water for further testing.

\subsection{Membrane characterization}

Contact angle measurements to evaluate the hydrophilicity of the membrane surface were performed with a KSV CAM 200 instrument (KSV Instruments, USA) using the sessile drop technique. Scanning Electron Microscopy (SEM, XL-30, Philips, Eindhoven, The Netherlands) equipment was used to study the morphology of the membrane crosssection. Due to the requirements of image magnification to determine the membrane pore size distribution, a Field Emission Gun Scanning Electron Microscope (FEG-SEM, FEI, The Netherlands) was used. Four samples of each type of doped membranes were analyzed to estimate the statistical pore size distribution and membrane surface porosity.

This method is based in the image processing of FEG-SEM pictures by using a threshold filter to identify the membrane pores as darker points onto the membrane surface. See reference [8].

Water uptake tests were conducted to evaluate the water sorption by the prepared membranes in order to determine the overall membrane porosity $\left(P_{r}\right)$ calculated with Eq. (1).

$P_{r}(\%)=\left(\frac{W_{w}-W_{d}}{S d \rho}\right) \times 100$

where $W_{w}$ and $W_{d}$ are the equilibrium weights of a membrane at swelling and dry states, respectively; $S$ is the membrane area; $d$ is the

Table 1

Composition and nomenclature of prepared membranes.

\begin{tabular}{llllll}
\hline Filler & Functionality & $\begin{array}{l}\text { PES } \\
\text { wt.\% }\end{array}$ & $\begin{array}{l}\text { Filler } \\
\text { wt.\% }\end{array}$ & $\begin{array}{l}\text { Solvent } \\
\text { wt.\% }\end{array}$ & Membrane \\
\hline None & None & 18 & 0.0 & 82.0 & 0 \\
SBA-15 & None & 18 & 0.6 & 81.4 & $\mathrm{~A}$ \\
SBA-15/CES-15 & $\mathrm{COOH}$ & 18 & 0.6 & 81.4 & $\mathrm{~B}$ \\
SBA-15-APTMS-15 & $\mathrm{NH}_{2}$ & 18 & 0.6 & 81.4 & $\mathrm{C}$ \\
SBA-15/AEAPTMS-15 & $\mathrm{NH}\left(\mathrm{CH}_{2}\right)_{2} \mathrm{NH}_{2}$ & 18 & 0.6 & 81.4 & $\mathrm{D}$ \\
& $\mathrm{NH}\left(\mathrm{CH}_{2}\right)_{2} \mathrm{NH}_{2}$ & 18 & 1.0 & 81.0 & $\mathrm{E}$ \\
SBA-15/CES-30 & $\mathrm{COOH}$ & 18 & 0.6 & 81.4 & $\mathrm{~F}$ \\
\end{tabular}

thickness and $\rho$ is the density of water. The membranes were immersed in water during $24 \mathrm{~h}$ prior to measurement of swelling state. The porosity data were the average values obtained for 4 samples of each membrane.

\subsection{Filtration experiments}

The membrane filtration performance was investigated by using a stainless steel cross-flow cell module having an effective membrane area of $0.005 \mathrm{~m}^{2}$ connected to a $2 \mathrm{~L}$ volume tank. The membrane permeability was determined in a filtration recycle mode for ultra-pure water (Milli-Q water).

In order to study the fouling mechanism, Bovine Serum Albumin (BSA) purchased from Sigma-Aldrich (St. Louis, USA) was selected as a model organic foulant in this study. The BSA solution $\left(0.2 \mathrm{~g} \mathrm{~L}^{-1}\right.$, $\mathrm{pH}$ 7.2) was prepared by using a $0.1 \mathrm{M}$ phosphate buffer solution (PBS). Initially, membrane compaction was performed for $2 \mathrm{~h}$ at $3 \mathrm{bar}$ of transmembrane pressure (TMP). After that, pure water and the protein solution filtration experiments were performed at 3 bar, $25 \pm$ $1{ }^{\circ} \mathrm{C}$, and $0.65 \mathrm{~m} \mathrm{~s}^{-1}$ cross-flow velocity. To study the effect of reversible and irreversible fouling mechanisms, the permeation of pure water was measured before and after studying the fouling resistance of membranes in contact with BSA solution following the protocol reported elsewhere [8]. To describe flux decline, the resistance-in-series model $[8,17]$ was used. In this model the water flux is written as a function of transmembrane pressure $(\Delta P)$, the viscosity $(\eta)$ and the total membrane resistance $\left(R_{t}\right)$.

When filtering pure water, the resistance against mass transport only involves the membrane intrinsic resistance $\left(R_{m}\right)$, whereas during filtration of a feed solution, the flux declines according to gel resistance layer formation $\left(R_{g}\right)$ and the solute adsorption of macromolecules (foulants) on membrane pore wall or surface $\left(R_{f}\right)$.

Membrane resistance values can be obtained from experimental data using the following equations:

$R_{m}=\frac{\Delta P}{\eta J_{w}^{i}}$

$R_{f}=\frac{\Delta P}{\eta J_{w}^{f}}-R_{m}$

$R_{g}=\frac{\Delta P}{\eta J_{v}}-\left(R_{m}+R_{f}\right)$

$R_{t}=R_{m}+R_{f}+R_{g}$ 
The flux of the feed solution is defined as $J_{v}$ and the pure water flux measured before and after the filtration experiments is represented as $J_{w}^{i}$ and $f_{w}^{f}$, respectively.

Membrane selectivity was explored in terms of BSA rejection (Eq. (6)) using a Cary 5000 UV-Vis-NIR Spectrophotometer to determine the permeate $\left(C_{p}\right)$ and feed $\left(C_{f}\right)$ concentrations of BSA.

$R(\%)=\left(1-\frac{C_{p}}{C_{f}}\right) \times 100$

\section{Results and discussion}

\subsection{Material characterization}

The hexagonal arrangement of mesostructured SBA-15-type materials has been studied by transmission electronic microscopy. TEM images of SBA-15 functionalized at lower organic content (Fig. 2a-d) evidenced the presence of an ordered $16 \mathrm{~mm}$ hexagonal structure. However, SBA-15/CES-30 sample (Fig. 2e and f) clearly showed areas of mesoporosity with low or non-mesoscopic ordering, which confirms the typical structure-distorting phenomena observed in functionalized mesostructured silica with high organic contents $[28,36]$.

Textural parameters deduced from nitrogen isotherms are shown in Table 2. It must be noted that the pore size values obtained by $\mathrm{N}_{2}$
Table 2

Textural properties and nitrogen content of synthesized silica particles.

\begin{tabular}{lllll}
\hline Sample & $\begin{array}{l}\mathrm{S}_{\mathrm{BET}} \\
\left(\mathrm{m}^{2} / \mathrm{g}\right)\end{array}$ & $\begin{array}{l}\mathrm{D}_{\mathrm{p}}{ }^{\mathrm{a}} \\
(\AA)\end{array}$ & $\begin{array}{l}\mathrm{V}_{\mathrm{t}}{ }^{\mathrm{a}} \\
\left(\mathrm{cm}^{3} / \mathrm{g}\right)\end{array}$ & $\begin{array}{l}\text { N content } \\
(\mathrm{mmol} / \mathrm{g})\end{array}$ \\
\hline SBA-15 & 620 & 84 & 0.96 & - \\
SBA-15/CES-15 & 561 & 79 & 0.83 & - \\
SBA-15-APTMS-15 & 579 & 81 & 0.87 & 2.0 \\
SBA-15/AEAPTMS-15 & 551 & 74 & 0.78 & 3.9 \\
SBA-15/CES-30 & 466 & 73 & 0.65 & - \\
\hline
\end{tabular}

a Pore size and total pore volume calculated by BJH method from the adsorption branch of the $\mathrm{N}_{2}$ isotherm.

b Nitrogen content by elemental analysis.

adsorption were in fair agreement with the measurements directly performed on TEM micrographs (Fig. 2).

All functionalized silica particles showed a decrease in textural properties compared to the pure SBA- 15 . These changes could be caused by the pore filling as a consequence of functionalization as well as the perturbation of organosilane molecules during the condensation process. These observed changes were more pronounced for higher loadings and among the samples with the same organic content for longer organosilane chains. Nevertheless, even the sample with the highest organic content, SBA-15/CES-30, displayed acceptable textural properties. The nitrogen content of the amino-functionalized samples is also shown in Table 2. As expected, the SBA-15/AEAPTMS to SBA-15/APTMS nitrogen content ratio is around two.
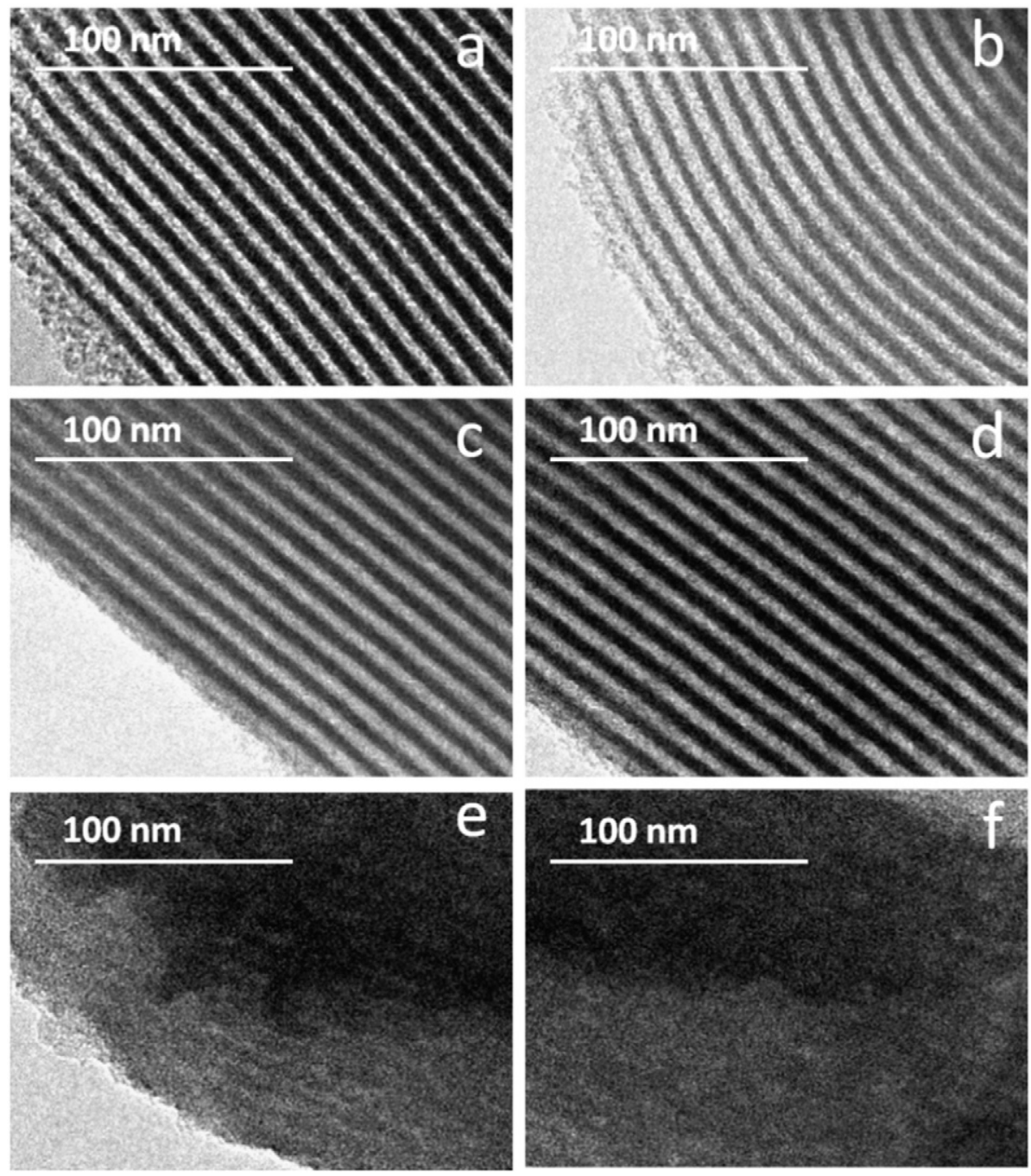

Fig. 2. TEM images of synthesized particles. (a) SBA-15. (b) SBA-15/CES-15. (c) SBA-15-APTMS-15. (d) SBA-15/AEAPTMS-15. (e and f) SBA-15/CES-30. 
The incorporation yield of the organic precursor has been evaluated by means of solid-state MAS-NMR. A single-pulse method was used for the NMR analysis of ${ }^{29} \mathrm{Si}$ nuclei, enabling the direct quantification of the different silicon species. Fig. 3 depicts the spectra corresponding to the synthesized materials, in which the different types of silicon environments are shown: $Q^{n}=\mathrm{Si}(\mathrm{OSi})_{n}(\mathrm{OX})_{4-n}$, wherein $n=2-4$ ( $Q^{2}$ at $-90 \mathrm{ppm}, Q^{3}$ at $-100 \mathrm{ppm}$ and $Q^{4}$ at $-110 \mathrm{ppm}$ ), and $T^{m}=$ $\mathrm{RSi}(\mathrm{OSi})_{m}(\mathrm{OX})_{3}-m$, wherein $m=2-3\left(T^{3}\right.$ at $-65 \mathrm{ppm}$ and $T^{2}$ at $-57 \mathrm{ppm}) . T^{m}$ signals reveal the presence of organic moieties chemically attached to the silica structure via $\mathrm{Si}-\mathrm{C}$ bonds, and hence can be used for the determination of actual organic incorporation. The resulting values are in fair agreement with those established (expected) in the synthesis procedure, which means an incorporation yield extent of about $90 \%$ in every case.

In order to determine the particle size distribution of the synthesized materials, stable dispersions of filler-solvent were prepared and analyzed by light scattering as shown in Fig. 4.

An increase of average particle size was observed as a result of functionalization of mesoporous silica SBA-15 particles with amine groups. This effect can be explained in terms of surface charge neutralization by protonation of amine groups on the particle surface. The negative charge of the deprotonated silanol groups is partially neutralized by the presence of positively charged amine functional groups. The colloidal stabilization was also negatively affected by the incorporation of added particles (Fig. 4c). As the number of particles increases in the colloidal suspensions the interaction between them is favored and, consequently, particle aggregates with bigger size are formed [17]. When the number of dispersed particles increases, the interaction between them is favored and agglomeration occurs through formation of larger particles with lower surface area to volume ratio. So, experimental results show that significant particle aggregation induced by interparticle attractive forces, such as van der Waals, is promoted with filler concentration.

\subsection{Membrane characterization}

A preliminary study was performed in order to explore the influence of pore ordering of silica particles on the morphology (Fig. 5a-b) and permeation (Fig. $5 \mathrm{c}$ ) of silica doped PES membranes. The morphology and permeation properties of the prepared membranes were quite different depending on the pore ordering of silica used as filler particles. Modified membranes with non-mesostructured silica exhibited a sponge-like structure in the macrovoid walls of its cross-section (Fig. 5a), while SBA-15 silica doped membranes showed thinner walls separating the macrovoid configuration (Fig. 5b). In addition,

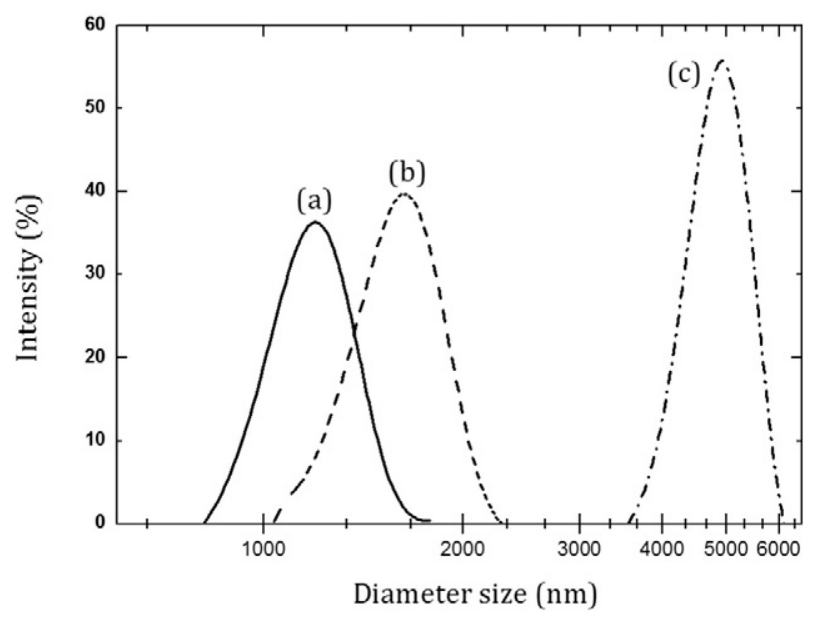

Fig. 4. Particle size distribution of fillers dispersed in NMP solvent. (a) SBA-15 in NMP at 0.6 wt.\%. (b) SBA-15/AEAPTMS-15 in NMP at 0.6 wt.\%. (c) SBA-15/AEAPTMS-15 in NMP at $1.0 \mathrm{wt} . \%$.

a thicker and denser top layer was observed in the morphology of non-mesostructured silica doped membranes (Fig. 5a). This fact could explain the lower water permeation obtained for this membrane. The thickness and porosity of the membrane upper layer mainly determine the permeation performance of the UF membranes (Fig. 5c). Taking into account the above results for further studies, the mesostructured silica particles were selected as fillers for ultrafiltration composite membrane preparation.

Membrane morphology was explored by SEM to observe the possible differences in the inner membrane structure of the prepared membranes from the cross-section images (Fig. 6). All the membranes prepared by the immersion-precipitation method exhibited an asymmetrical configuration with a distribution of open macrovoids in its structure. No appreciable differences were observed for both the size and distribution of macrovoids for the studied membranes. Some factors could contribute to this similar inner morphology: the polymer content used for polymeric solution preparation (18 wt.\%), the low concentration of particle doping proposed (0.6 wt.\%), and the instantaneous immersion of polymeric film after the casting procedure without any solvent evaporation time, previous to the demixing process. The presence of fillers into the membrane structure was confirmed at higher magnification as shown in Fig. 6d, where SEM and inserted TEM images are illustrated.

The porosity of the prepared membranes was studied in terms of surface and overall porosities by FEG-SEM and water uptake experiments,
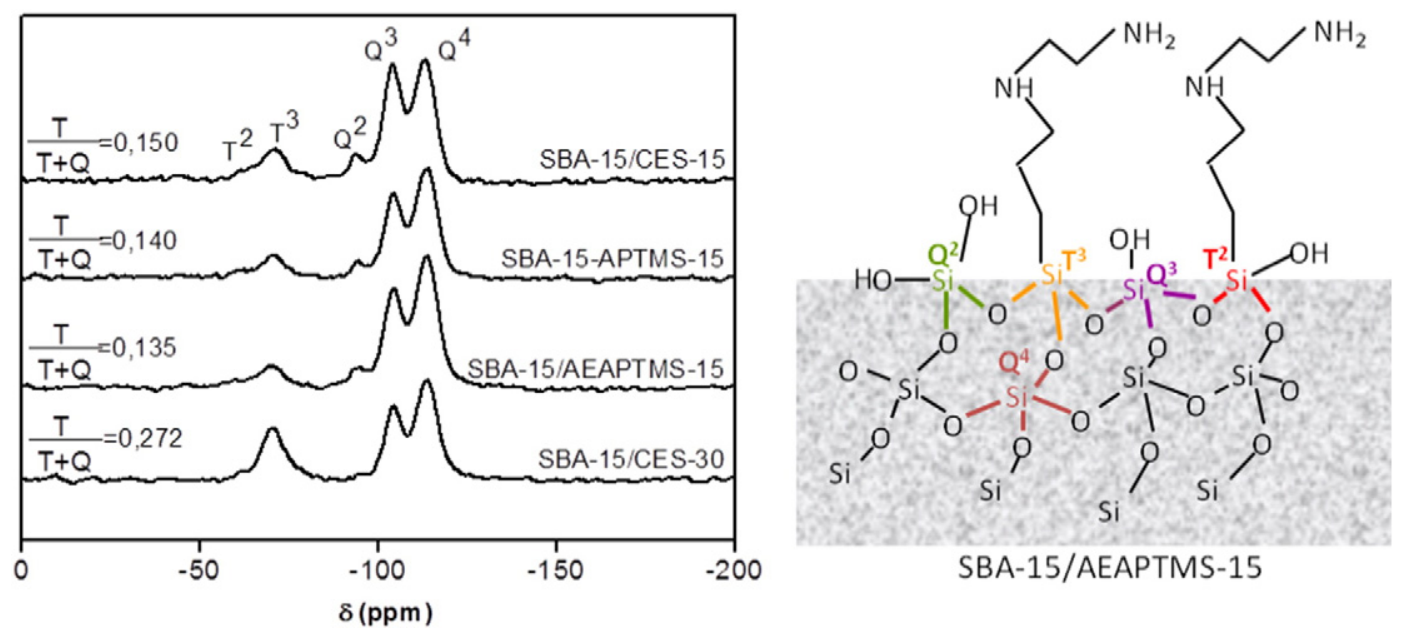

Fig. 3. ${ }^{29}$ Si MAS-NMR spectra corresponding to the functionalized samples. 

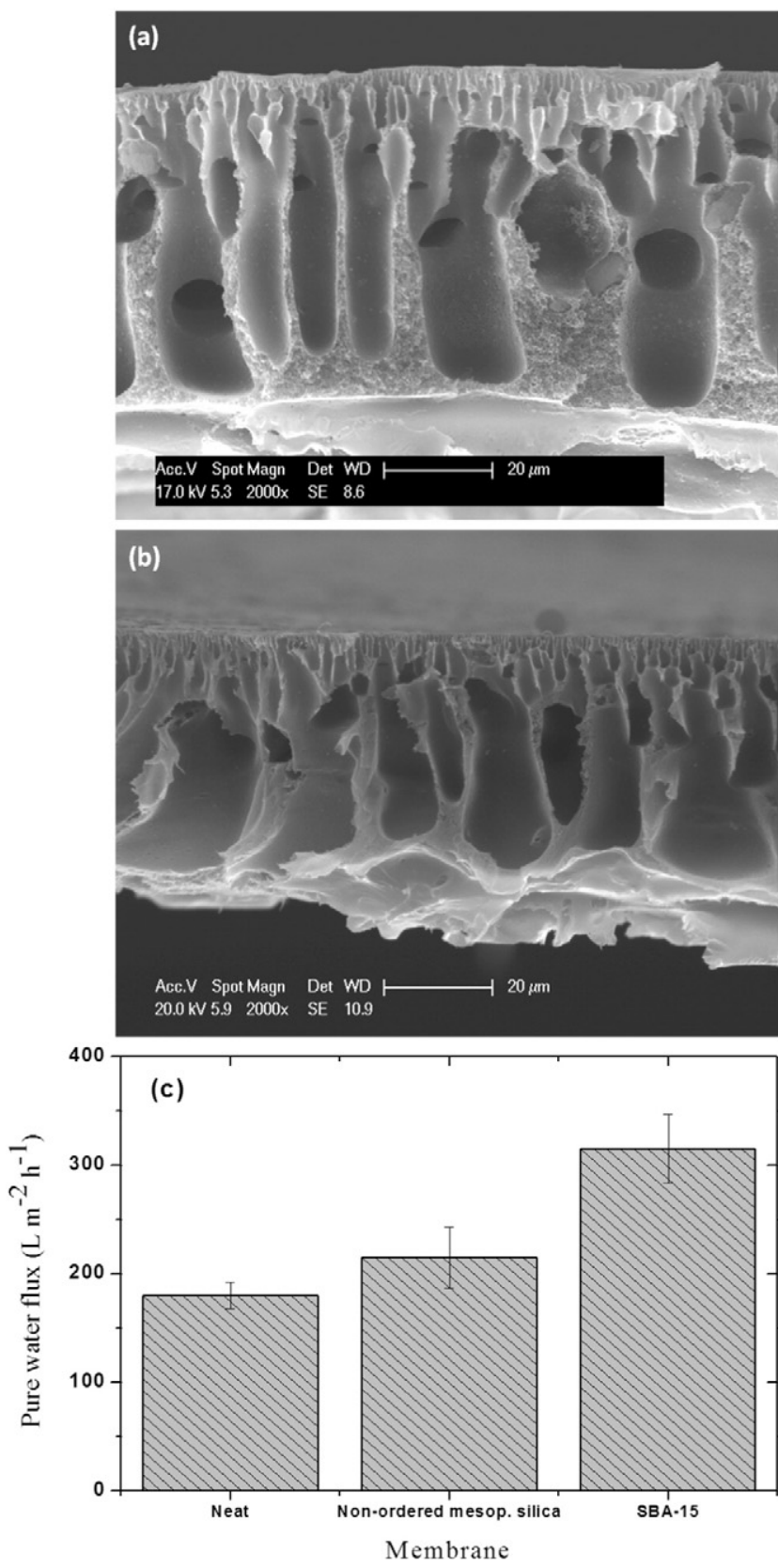

Fig. 5. SEM cross-section images and water permeation of membranes. (a) Membrane doped with non-ordered mesoporous silica. (b) Membrane doped with mesostructured silica (SBA-15). (c) Membrane water permeation.

respectively (Table 3 ). In addition, the hydrophilicity of the prepared membranes was evaluated by measuring the tangent (angle) of water drop with the membrane surface. Contact angle measurements are also summarized in Table 3.

Data listed in Table 3 confirmed that the addition of mesostructured silica particles to the membrane composition brought about an increase of the porosity and an enhancement of the hydrophilicity at the same time. A similar behavior was observed for membranes B and C doped with fillers functionalized with carboxylic acid and amine groups, respectively. The overall porosity of the membranes, determined by the free-volume of membrane structure occupied by absorbed water, was calculated by means of water uptake measurements. The water transport through the membrane can be regulated by two factors: porosity and hydrophilicity of polymer matrix. A porous membrane with high pore density in its surface and good connectivity between surface and inner (macrovoids) pores supplies the water transport through the membrane. Thus, considering the similarity of macrovoid dimensions of the tested membranes (Fig. 6), the water absorption should be determined by the surface porous characteristics: size and number of pores. The incorporation of hydrophilic particles into the polymeric solution helps in the formation of higher number of polymer-lean phase zones into the polymer matrix during the membrane synthesis process, promoting the formation of higher pore number. Some authors have indicated that hydrophilic particles used for composite membrane preparation can be considered as pore formation agents [37,38]. The enhanced pore formation could be caused by two effects: the hindrance effect of particles between the polymer chains into the polymer matrix configuration and the great affinity of fillers to water that increases the water diffusion during the demixing process [17].

The decrease of contact angle can be explained by the combined effect of incorporation of hydrophilic fillers near the membrane surface and formation of higher surface pore. The presence of selected nanocharges near the membrane surface promotes the development of an upper membrane layer more hydrophilic as a consequence of higher water affinity of doping particles. Moreover, the hydrophilic character of particles as determined by the particle-water interaction extent could be explained in terms of active hydrophilic center abundance in the chemical structure of particles. This fact could clarify the observed decrease in the contact angle measurements for membranes doped with particles with amine-reach functional groups. In addition, more water can be absorbed by the membrane surface during the contact angle experiments due to the increase in pore size. Hydrophilic particle incorporation increases water diffusion into the growing membrane whereas the interaction between polymer and solvent molecules is negatively affected by the hindrance effect of fillers. As the precipitation rate decreases the two phases have more time to separate and the size of the pore enlarges. Higher particle contents (Memb. F, 1 wt.\%) lead to formation of membranes with less porosity instead of promoting a more porous structure. This effect could be associated with the formation of aggregates (Fig. 4c) that decreases the number of free particles, affecting negatively the pore formation. Moreover, a higher content of fillers could lead to the pore plugging, causing a decrease on the membrane permeation rate $[17,39]$.

The membrane D, doped with SBA-15/AEAPTMS-15 exhibited the highest porosity and hydrophilicity. The functionalization of SBA-15 with AEAPTMS organic precursor leads to the incorporation of organic chains with two fold amine groups in its chemical composition and larger chain length in comparison with the amine group source SBA-15-APTMS15 (Fig. 1b and c). As the number of active (hydrophilic) sites increases, the interaction with water molecules via hydrogen bonding is statistically promoted, reinforcing the water transport through the membrane. In addition, considering the chain length of the added functional group, the active sites could be more accessible to hydrogen bonding interaction.

Membrane F, doped in around 30 wt.\% with carboxylic groups, showed a higher porous structure and hydrophilicity than membrane $\mathrm{B}$; however, its performance was poorer than membrane D. And this was also found for membrane $\mathrm{E}$ in spite of its higher filler content ( $1 \%$ against $0.6 \%$ ). This behavior confirms previous reports suggesting that low filler loading can lead to better membrane performance [17].

In addition, the effect of ordered mesoporous silica particle incorporation on the membrane surface morphology was also explored by FEGSEM to determine the surface pore size distribution of the prepared membranes (Fig. 7).

It could be observed that addition of particles to the composition of polymeric solution increased the membrane pore size mode from 6 to $8 \mathrm{~nm}$. This behavior could be explained in terms of size increase of the polymer-lean phase zones into the polymer matrix due to the hindrance effect of particles, which disturbs the polymer chain interaction, during the membrane formation. It is also remarkable the transition from quite unimodal distribution for neat membrane to wide-spread distribution for membrane $C$. This effect could be associated with the wide particle size distribution obtained on measuring of fillers (Fig. 4). Conversely, 

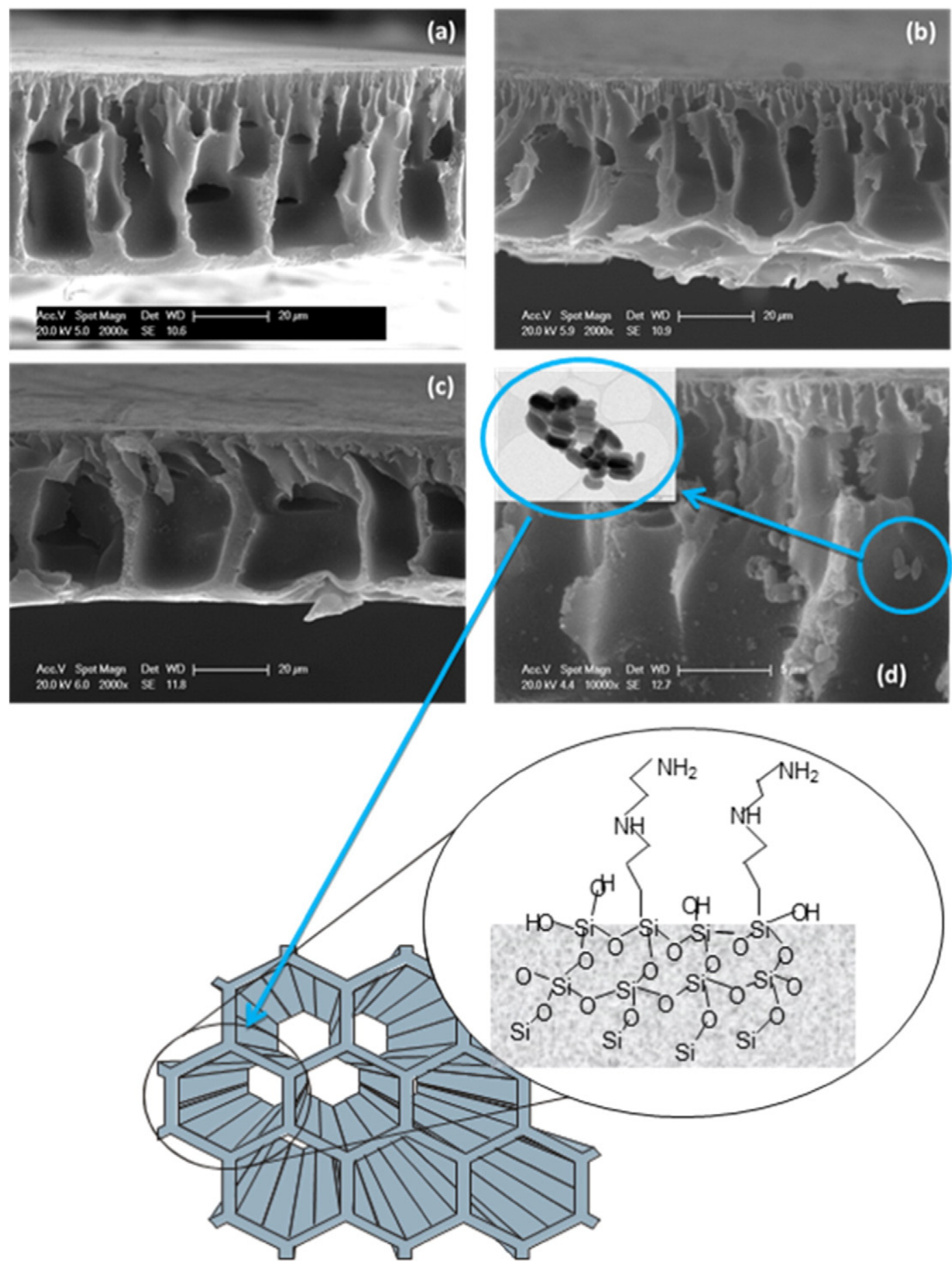

Fig. 6. SEM cross-section images of manufactured membranes. (a) Membrane 0. (b) Membrane A. (c) Membrane C. (d) Membrane D, at higher magnification.

Table 3

Values obtained from the porosity and hydrophilicity characterization of prepared membranes.

\begin{tabular}{llll}
\hline Membrane & $\begin{array}{l}\text { Surface pore density } \\
\text { (pore number } \mu \mathrm{m}^{-2} \text { ) }\end{array}$ & $\begin{array}{l}\text { Overall porosity } \\
(\%)\end{array}$ & $\begin{array}{l}\text { Contact angle } \\
\left({ }^{\circ}\right)\end{array}$ \\
\hline 0 & $32.4 \pm 0.3$ & $69.1 \pm 1.5$ & $70.3 \pm 0.8$ \\
A & $38.7 \pm 0.2$ & $77.4 \pm 1.4$ & $64.7 \pm 0.9$ \\
B & $43.1 \pm 0.3$ & $78.0 \pm 1.3$ & $63.0 \pm 0.7$ \\
C & $42.4 \pm 0.4$ & $81.6 \pm 1.8$ & $61.7 \pm 1.0$ \\
D & $93.2 \pm 0.3$ & $97.4 \pm 2.8$ & $55.0 \pm 1.2$ \\
E & $62.7 \pm 0.3$ & $76.3 \pm 2.2$ & $57.2 \pm 1.4$ \\
F & $59.7 \pm 0.3$ & $82.6 \pm 2.8$ & $58.1 \pm 1.1$ \\
\hline
\end{tabular}

the addition of functionalized silica particles with double amine groups exceedingly enhanced the number of pores formed onto the membrane surface (membrane D); however the statistical pore size distribution was not significantly modified. It is difficult at this point to find a reliable explanation for these effects. Perhaps, the long organic chains ensure the full accessibility of the non-solvent molecules (water) to the amine hydrophilic sites during the first stage of membrane formation.

\subsection{Membrane permeation and fouling performance}

The effect of particle addition on the membrane permeability is shown in Fig. 8. The water permeation flux increased upon incorporating silica 

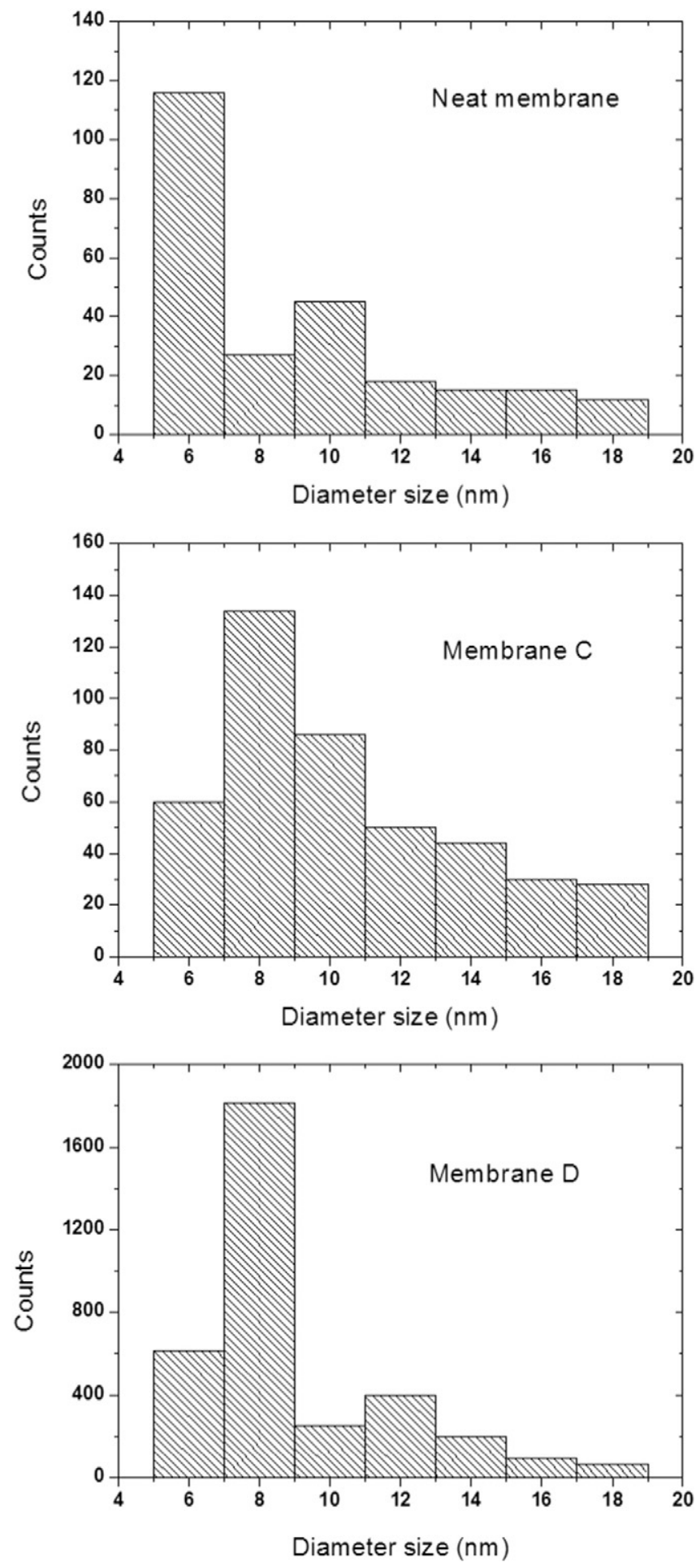

Fig. 7. Surface pore size distribution of manufactured membranes.

fillers from $180 \mathrm{~L} \mathrm{~m}^{-2} \mathrm{~h}^{-1}$ to $315 \mathrm{~L} \mathrm{~m}^{-2} \mathrm{~h}^{-1}$ for membrane A and up to $631 \mathrm{~L} \mathrm{~m}^{-2} \mathrm{~h}^{-1}$ for membrane $\mathrm{D}$. This observed increase is higher than literature reports referring non-mesostructured silica particles as hydrophilic fillers [19-22]. The ordered configuration of mesopores guaranties a better accessibility of water molecules to the active sites of silica functional groups. A higher hydrophilicity reinforces the diffusional exchange between solvent and non-solvent, which leads to a lower polymer concentration at the interphase between polymer solution and non-solvent bath during immersion-precipitation process and hence to a higher porosity and enhanced permeability of the membrane. Indeed, the

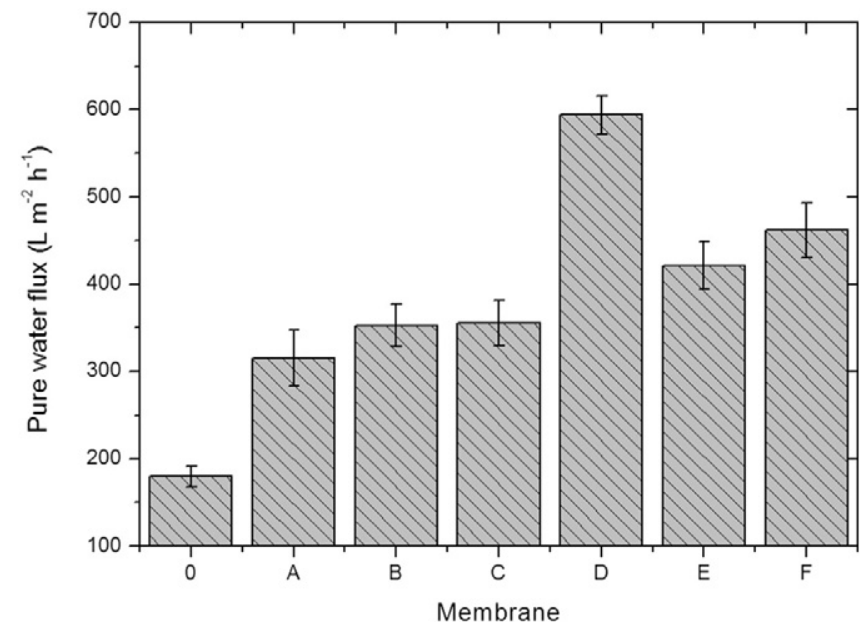

Fig. 8. Effect of membrane chemical composition on the pure water permeation. Error bars give the standard deviation.

permeation potential of membranes is in very good agreement with the experimental values of porosity, pore size and hydrophilicity found for the tested membranes (Table 3 ). As mentioned above, the increase both of doping amount (membrane F) and percentage of amine functionalization (membrane E) does not lead to further improvement of membrane permeation.

The membrane fouling performance was studied considering the different contributions of membrane resistance to water permeation: intrinsic, reversible, irreversible and total resistances (Fig. 9). Total membrane resistance is calculated as the sum of all contributions. BSA $0.2 \mathrm{~g} \mathrm{~L}^{-1}$ aqueous solution was selected as feed water to estimate the reversible and irreversible fouling contributions during the membrane water permeation [17]. The intrinsic resistance is determined from pure water permeate flux as the inherent opposition to water transport, which is mainly governed by the membrane morphology. The intrinsic resistance contribution for doped membranes was around 50\% of the total resistance in comparison with the $38 \%$ observed for the neat PES membrane.

Fouling mechanisms can be reversible or irreversible. Reversible fouling is mainly caused by the formation of a cake layer (deposition, gel formation) or concentration polarization of foulants onto the membrane surface. It can be removed by an appropriate physical washing such as backwashing or surface washing. Conversely, irreversible fouling is caused by adsorption and/or pore blockage $[37,39]$.

In other to determine the contributions to flux decline of irreversible and reversible fouling mechanisms, the tested membranes were

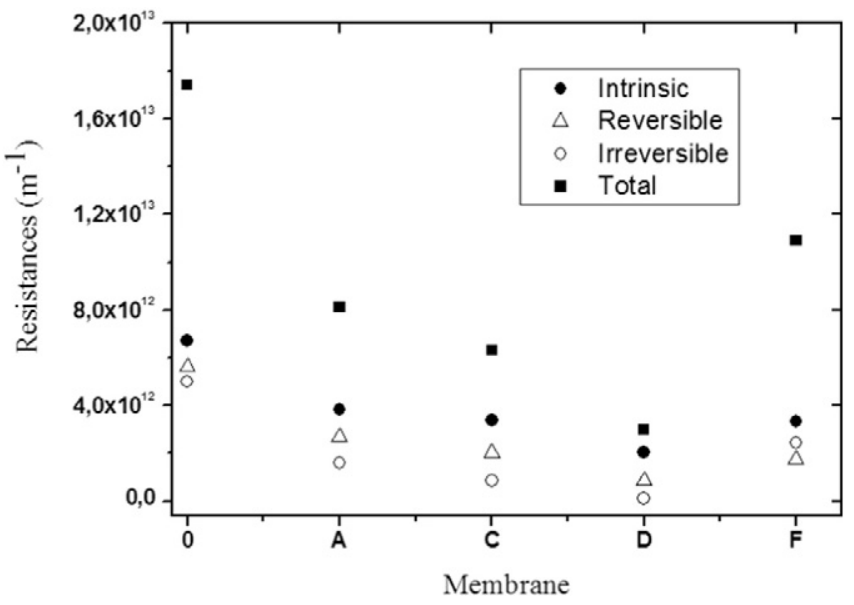

Fig. 9. Fouling resistances for neat and doped prepared membranes. 
washed for $2 \mathrm{~h}$ with deionized water as membrane cleaning procedure. Subsequently, the pure water flux was measured in order to estimate the reversible fouling degree. The irreversible fouling resistance is determined by measuring the BSA solution flux and subtracting the contribution of intrinsic resistance.

The extent of reversible and irreversible fouling significantly decreased as result of silica particle incorporation into the polymeric solution. The effect of hydrophilic character of mesostructured silica particles is responsible for interaction mitigation of foulants with the polymer matrix, resulting in higher membrane permeation due to the increase of hydrophilicity of the membrane surface and pore walls. Particularly, for membrane D, fouling degree was reduced around 75 and $95 \%$ for reversible and irreversible fouling resistances respectively, in comparison with the neat PES membrane.

For all of the tested membranes the rejection potential was almost $100 \%$, so it can be affirmed that the trade-off between selectivity and permeation was improved, increasing the permeate flux while the rejection was hardly affected.

Finally, membrane permeation stability was evaluated by measuring the permeate flux of the best membrane (membrane $\mathrm{D}$ ) in consecutive cycles of BSA solution filtration. The membrane was cleaned after each cycle with distilled water for $2 \mathrm{~h}$. As it can be observed in Fig. 10 the permeate flux decreased slightly after the first cycle probably due to the reversible fouling. However, in the rest of the cycles the permeate flux was maintained constant, showing a good stability as a guarantee of the integrity of membrane functions.

\section{Conclusions}

In this work a wide characterization of synthesized particles used as fillers and the corresponding modified PES membranes has been accomplished. The more relevant conclusions of this study are abbreviated as follows:

Mesostructured silica SBA-15 functionalized with amine and carboxylic groups was successfully prepared by co-condensation in the expected concentration value.

Morphology of doped membranes, in terms of porosity and pore size distribution, significantly changed as a result of the ordered mesoporous silica particle incorporation, especially for membranes doped with SBA-15 functionalized with diamine chains.

As a result of surface porosity and hydrophilicity improvement of the doped membranes, water permeation significantly increased in all cases as compared to pure PES membranes, but very especially for the membrane denoted as $\mathrm{D}$. It seems that the pore ordering preservation of the mesoporous silica particles used in this study is the

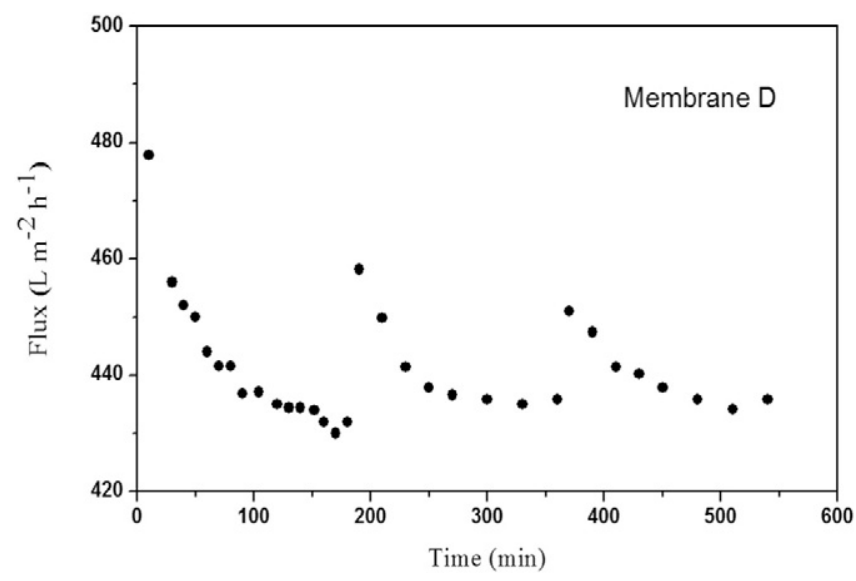

Fig. 10. Permeate flux of three different cycles of BSA solution filtration for PES membrane containing SBA-15/AEAPTMS-15 filler. guarantee for a better interaction between the water stream and the hydrophilic particle functional groups during membrane permeation. The antifouling property of the modified membranes was improved, enhanced especially against irreversible fouling, without negatively affecting the protein rejection potential of membranes.

Finally, the novel ultrafiltration doped membranes prepared in this investigation exhibited a stable permeation performance during repeated stability tests.

\section{Acknowledgments}

The authors thank the financial support of the Spanish Government provided through CTQ2011-22707 and MAT2013-45071 projects and the Regional Government of Madrid (S-0505/AMB/0395) provided through project REMTAVARES.

\section{References}

[1] J.I. Moch, Membranes, Hollow Fiber, Kirk-Othmer Encyclopedia of Chemical Technology, 162005. 1-31.

[2] M.M. Pendergast, E.M.V. Hoek, A review of water treatment membrane nanotechnologies, Energy Environ. Sci. 4 (2011) 1946-1971.

[3] A.V.R. Reddy, D.J. Mohan, A. Bhattacharya, V.J. Shah, P.K. Ghosh, Surface modification of ultrafiltration membranes by preadsorption of a negatively charged polymer. Permeation of water soluble polymers and inorganic salt solutions and fouling resistance properties, J. Membr. Sci. 214 (2003) 211-221.

[4] K.J. Howe, M.M. Clark, Fouling of microfiltration and ultrafiltration membranes by natural waters, Environ. Sci. Technol. 36 (2002) 3571-3576.

[5] R.W. Baker, Membrane Technology and Applications, Second ed. John Wiley \& Sons, California, 2004.

[6] J. Kim, B. Van der Bruggen, The use of nanoparticles in polymeric and ceramic membrane structure: review of manufacturing procedures and performance improvement for water treatment, Environ. Pollut. 158 (2010) 2335-2349.

[7] T.H. Bae, T.M. Tak, Effect of $\mathrm{TiO}_{2}$ nanoparticles on fouling mitigation of ultrafiltration membranes for activated sludge filtration, J. Membr. Sci. 249 (2005) 1-8.

[8] J.M. Arsuaga, A. Sotto, G. del Rosario, A. Martínez, S. Molina, S.B. Teli, J. de Abajo, Influence of the type, size, and distribution of metal oxide particles on the properties of nanocomposite ultrafiltration membranes, J. Membr. Sci. 428 (2013) 131-141.

[9] Y. Mansourpanah, S.S. Madaeni, A. Rahimpour, A. Farhadian, A.H. Taheri, Formation of appropriate sites on nanofiltration membrane surface for binding $\mathrm{TiO}_{2}$ photocatalyst: performance, characterization and fouling-resistant capability, J. Membr. Sci. 330 (2009) 297-306

[10] J. Kim, A. Sotto, J. Chang, A. Boromand, B. Van Der Bruggen, Embedding $\mathrm{TiO}_{2}$ nanoparticles versus surface coating by layer-by-layer deposition on nanoporous polymeric films, Microporous Mesoporous Mater. 173 (2013) 121-128.

[11] A. Sotto, J. Kim, J.M. Arsuaga, G. del Rosario, A. Martínez, D. Nam, P. Luis, B. Van Der Bruggen, Binary metal oxides for composite ultrafiltration membranes, J. Mater. Chem. A 2 (2014) 7054-7064.

[12] L.Y. Yu, Z.-L. Xu, Hong-Mei Shen, Hu Yang, Preparation and characterization of PVDF-SiO ${ }_{2}$ composite hollow fiber UF membrane by sol-gel method, J. Membr. Sci. 337 (2009) 257-265.

[13] S. Balta, A. Sotto, P. Luis, L. Benea, B. Van der Bruggen, J. Kim, A new outlook on membrane enhancement with nanoparticles: the alternative of $\mathrm{ZnO}$, J. Membr. Sci. 389 (2012) 155-161.

[14] N. Maximous, G. Nakhla, W. Wan, K. Wong, Preparation, characterization and performance of $\mathrm{Al}_{2} \mathrm{O}_{3} / \mathrm{PES}$ membrane for waste water filtration, J. Membr. Sci. 341 (2009) 67-75.

[15] J. Huang, G. Arthanareeswaran, K. Zhang, Effect of silver loaded sodium zirconium phosphate (nanoAgZ) nanoparticles incorporation on PES membrane performance, Desalination 285 (2012) 100-107.

[16] C. de Lannoy, E. Soyer, M.R. Wiesner, Optimizing carbon nanotube-reinforced polysulfone ultrafiltration membranes through carboxylic acid functionalization, J. Membr. Sci. 447 (2013) 395-402.

[17] A. Sotto, A. Boromand, R. Zhang, P. Luis, J.M. Arsuaga, J. Kim, B. Van der Bruggen, Effect of nanoparticle aggregation at low concentrations of $\mathrm{TiO}_{2}$ on the hydrophilicity, morphology, and fouling resistance of PES-TiO2 membranes, J. Colloid Interface Sci. 363 (2011) 540-550.

[18] J. Dulebohn, P. Ahmadiannamini, T. Wang, S.S. Kim, T.J. Pinnavaia, V.V. Tarabara, Polymer mesocomposites: ultrafiltration membrane materials with enhanced permeability, selectivity and fouling resistance, J. Membr. Sci. 453 (2014) 478-488.

[19] L.J. Zhu, L.P. Zhun, J.H. Jiang, Z. Yi, Y.F. Zhao, B.K. Zhu, Y.Y. Xu, Hydrophilic and anti-fouling polyethersulfone ultrafiltration membranes with poly(2hydroxyethylmethacrylate) grafted silica nanoparticles as additive, J. Membr. Sci. 451 (2014) 157-168.

[20] K. Wang, Z. Xie, B. Ladewig, H. Wang, J. Huang, K. Zhang, Fabrication of polyethersulfone-mesoporous silica nanocomposite ultrafiltration membranes with antifouling properties, J. Membr. Sci. 423-424 (2012) 362-370.

[21] Y. Zhanga, Z. Jin, J. Sunarso, J. Li, Development of nonstoichiometric silica with multiactive groups/polysulfone composite membranes for wastewater containing oil, Chem. Eng. J. 170 (2011) 14-20. 
[22] G. Arthanareeswaran, T.K. Sriyamuna Devi, M. Raajenthiren, Effect of silica particles on cellulose acetate blend ultrafiltration membranes: part I, Sep. Purif. Technol. 64 (2008) 38-47.

23] L. Cao, T. Man, M. Kruk, Synthesis of ultra-large-pore SBA-15 silica with twodimensional hexagonal structure using triisopropylbenzene as micelle expander, J. Chem. Mater. 21 (2009) 1144-1153.

[24] M. Manzano, M. Vallet-Regí, New developments in ordered mesoporous materials for drug delivery, J. Chem. Mater. 20 (2010) 5593-5604.

[25] X.G. Wang, S.K.L. Kyle, J.C.C. Chan, S.F. Cheng, Direct synthesis and catalytic applications of ordered large pore aminopropyl-functionalized SBA-15 mesoporous materials, J. Phys. Chem. B 109 (2004) 1763-1769.

[26] F. Zheng, D.N. Tran, B. Busche, G. Fryxell, R.S. Addleman, T.S. Zemanian, C.L. Aardahl, Ethylenediamine-modified SBA-15 as regenerable $\mathrm{CO}_{2}$ sorbent, Ind. Eng. Chem. Res. 44 (2005) 3099-3105.

[27] J.M. Rosenholm, A. Penninkangas, M. Lindén, Amino functionalization of large pore mesoscopically ordered silica by a one-step hyperbranching polymerization of a surface grown polyethyleneimine, Chem. Commun. 37 (2006) 3909-3911.

28] Q. Tao, Z. Xu, J. Wang, F. Liu, H. Wan, S. Zheng, Adsorption of humic acid to aminopropyl functionalized SBA-15, Microporous Mesoporous Mater. 131 (2010) 177-185.

[29] J. Aguado, J.M. Arsuaga, A. Arencibia, M. Lindo, V. Gascon, Aqueous heavy metals removal by adsorption on amine-functionalized mesoporous silica, J. Hazard. Mater. 163 (2009) 213-221.

[30] C.T. Tsai, Y.C. Pan, C.C. Ting, S. Vetrivel, A.S.T. Chiang, G.T.K. Feyb, H.M. Kao, A simple one-pot route to mesoporous silicas SBA-15 functionalized with exceptionally hig loadings of pendant carboxylic acid groups, Chem. Commun. 33 (2009) 5018-5020.
[31] L. Han, O. Terasaki, S. Che, Carboxylic group functionalized ordered mesoporous silicas, J. Mater. Chem. 21 (2011) 11033-11039.

[32] F. Hoffmann, M. Cornelius, J. Morell, M. Froba, Silica-based mesoporous organicinorganic hybrid materials, Angew. Chem. Int. 45 (2006) 3216-3251.

[33] M.H. Lim, A. Stein, Comparative studies of grafting and direct syntheses of inorganic-organic hybrid mesoporous materials, J. Chem. Mater. 11 (1999) 3285-3295.

[34] N. García, E. Benito, J. Guzmán, P. Tiemblo, V. Morales, R.A. García, Functionalization of SBA-15 by an acid-catalyzed approach: a surface characterization study, Microporous Mesoporous Mater. 106 (2007) 129-139.

[35] D. Zhao, J. Feng, Q. Huo, N. Meloshz, G.H. Fredrikson, B.F. Chmelka, G.D. Stucky, Triblock copolymer syntheses of mesoporous silica with periodic 50 to 300 angstrom pores, Science 279 (1998) 548-552.

[36] D. Margolese, J.A. Melero, S.C. Christiansen, B.F. Chmelka, G.D. Stucky, Direct syntheses of ordered SBA-15 mesoporous silica containing sulfonic acid groups, J. Chem. Mater. 12 (2000) 2448-2459.

[37] A. Rahimpour, S.S. Madaeni, A.H. Taheri, Y. Mansourpanah, Coupling $\mathrm{TiO}_{2}$ nanoparticles with UV irradiation for modification of polyethersulfone ultrafiltration membranes, J. Membr. Sci. 313 (2008) 158-169.

[38] Y. Yang, H. Zhang, P. Wang, Q. Zheng, J. Li, The influence of nano-sized $\mathrm{TiO}_{2}$ fillers on the morphologies and properties of PSF UF membrane, J. Membr. Sci. 288 (2007) 231-238.

[39] A. Razmjou, J. Mansouri, V. Chen, The effects of mechanical and chemical modification of $\mathrm{TiO}_{2}$ nanoparticles on the surface chemistry, structure and fouling performance of PES ultrafiltration membranes, J. Membr. Sci. 378 (2011) 73. 